\title{
Safe Return to Exercise after COVID-19 Infection
}

\author{
*Angelo V. Vasiliadis ${ }^{1}$ and Vasiliki Boka ${ }^{2}$
}

\begin{abstract}
COVID-19 has been posing a significant challenge to the athletic community. A wide spectrum of clinical features was reported in athletes with confirmed COVID-19 infection having mild to severe symptoms requiring hospitalisation and intensive medical care. Possibly, a lack of exercise may negatively impact the immune system, leading to an increased susceptibility to infection and comorbidities. Determining safe return to exercise after COVID-19 infection is a challenge for sports physicians. A gradual return to exercise under the guidance of a specialised medical team to achieve the pre-infection fitness level is of utmost importance. Individualised exercise programmes must be initiated at low intensity which could be gradually made more rigorous based on the pertinent metabolic equivalent. Thus, there is a clear need for clinical recommendations before athletes may return to exercise after COVID-19 infection.
\end{abstract}

Keywords: COVID-19; Coronavirus; Exercise; Athletes; Return to Sports.

$\mathrm{I}$ N DECEMBER 2019, THE WORLD HEALTH Organization (WHO) was informed about cases of pneumonia detected in the city of Wuhan in China. By the first week of January 2020, the presence of a novel coronavirus which became known as COVID-19 was officially announced by Chinese authorities. Approximately 10 days into the month of March 2020, the WHO declared the COVID-19 outbreak as a global pandemic. ${ }^{1}$ In less than a year, the COVID-19 pandemic emerged as a worldwide health issue with over 40 million documented cases and 1.1 million deaths worldwide. ${ }^{2}$ As the war against this pandemic continues, the world of sports faces the most significant health battle it has ever faced in modern history. Although athletes - fulfilling the following three criteria: (i) intent of the exercise, (ii) participation in sport events and (iii) registration in a sports federation are not considered to be at higher risk of COVID-19 infection, they may still be at risk of acquiring a COVID-19 infection. ${ }^{3,4}$ As expected, a significant number of athletes have been affected by COVID-19 infection or have been in close contact with somebody who has tested positive. ${ }^{5,6}$ Athletes need to follow the infection prevention strategies suggested for the general population including self-isolation for the entire symptomatic period to curtail the spread of the coronavirus and protect themselves, their families and their communities. ${ }^{6}$

The public health recommendations such as the universal closure of stadiums, gymnasiums and fitness centres and the need for quarantine or self-isolation (for a period of two weeks) of athletes who tested positive are fundamental. ${ }^{6,7}$ These recommendations are aimed at curtailing the spread of the disease and protect individuals and their communities. However, they also led to the disruption of many regular life activities, including physical activity in general and sports in particular. These drastic lifestyle changes resulting from self-isolation and physical inactivity have increased the risk of negative, acute and, possibly, long-term consequences for the athletes' health. ${ }^{7,8}$ There are concerns that the said lack of exercise routines and engagement in sports activities may have a serious impact on the immune system and the physical and mental health of athletes, leading also to the exacerbation of pre-existing diseases or development of new diseases. ${ }^{7}$

\section{THE PROBLEM}

Physical inactivity, defined as the lack of sufficient daily physical activity, and disruption of exercise routines may increase susceptibility to infections, whether being an athlete or not, due to dysfunction or deficiency of components of the immune system and also increase the overall risk of developing additional comorbidities over time. ${ }^{7}$ Moreover, the reduction of physical activity may negatively impact an athlete's physical fitness due to the loss of skeletal muscle mass and a concomitant decline in muscle strength., ${ }^{910}$ The negative metabolic effects of physical inactivity have been previously described. ${ }^{7,9}$ For instance, low levels of ambulatory activity for two weeks (a reduction in the number of daily steps from 10,000 to 1,000 ) may initiate or contribute to metabolic alterations, including reduced peripheral insulin sensitivity, impaired lipid profile and increased deposition of visceral fat. ${ }^{8,11}$ The decrease in the physical activity of a COVID-19-positive athlete during self-isolation has been documented. Each athlete responds and recovers differently and may face difficulties as they return to 


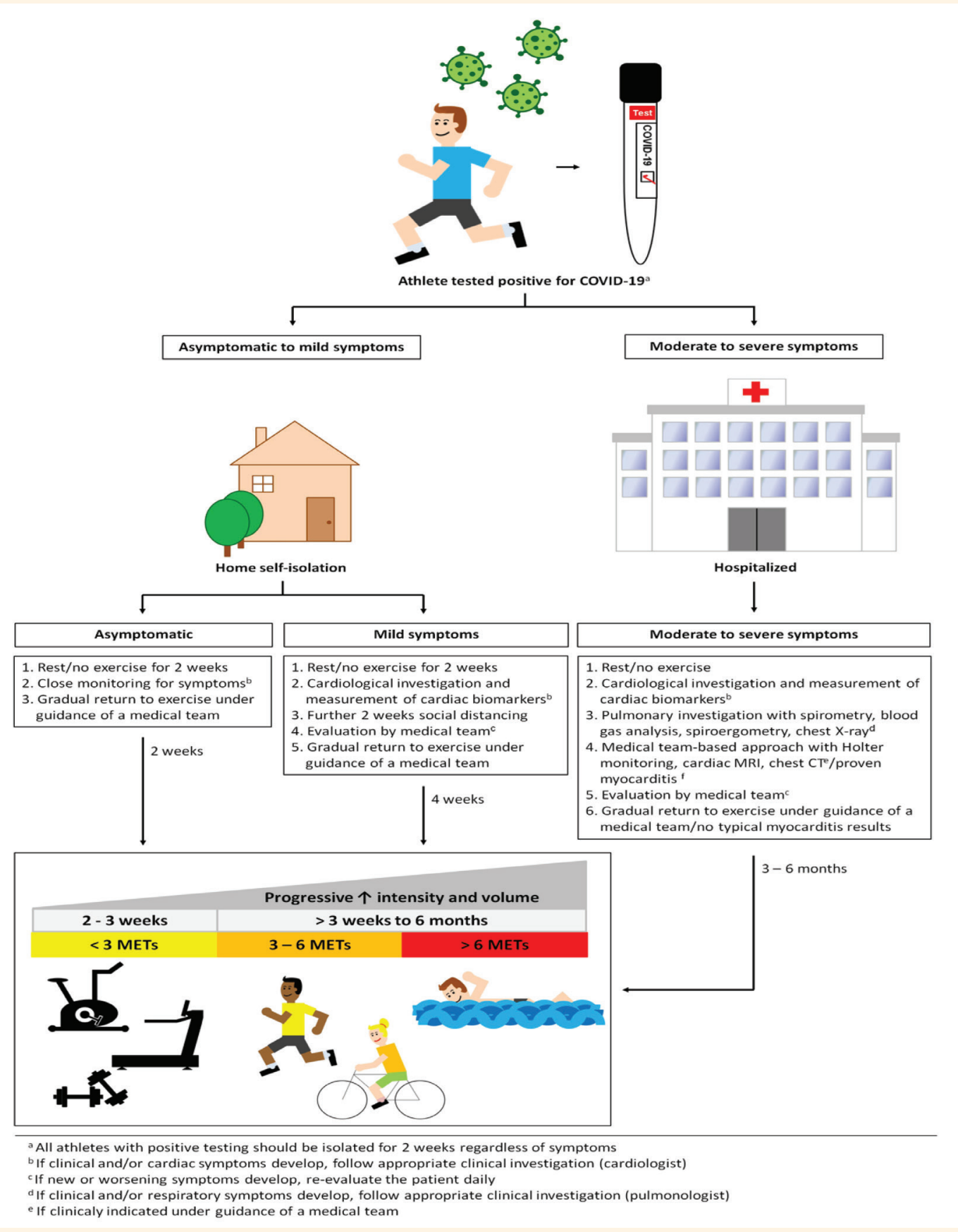

Figure 1: COVID-19 return-to-exercise algorithm for athletes.

$M R I=$ magnetic resonance imaging; $C T$ = computed tomography; METs = metabolic equivalents.

activities of daily living and exercise. ${ }^{7}$ It is, therefore, critical to guide an athlete diagnosed with COVID-19 for their safe return to exercise.

\section{GENERAL RECOMMENDATIONS FOR ATHLETES WITH COVID-19}

In this regard, a panel of international experts has classified athletes who have been diagnosed with COVID-19 infection into five different groups as follows: (i) asymptomatic; (ii) those with mild symptoms; (iii) those with moderate symptoms; (iv) those with severe symptoms without mechanical ventilation; and (v) those with severe symptoms with mechanical ventilation and/or myocardial injury. ${ }^{5,6,8}$ They have also provided specific recommendations for each group on how to safely return to exercise. ${ }^{5,6,8}$ While a majority of the athletes with COVID-19 infection either remain asymptomatic or experience mild symptoms, a comprehensive clinical evaluation is recommended before they return to exercise. Specifically, two-weeks of self-isolation and rest from exercise followed by a gradual return to exercise under 


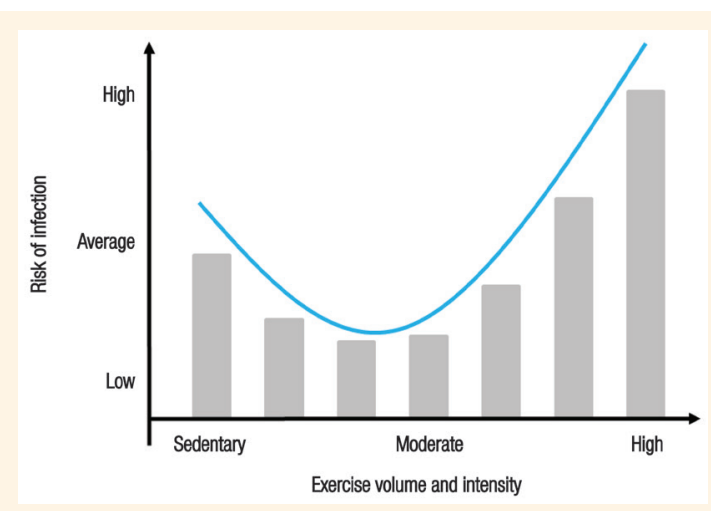

Figure 2: J-shaped curve of the relationship between the exercise volume and intensity and risk of viral infection.

the guidance of a medical team have been proposed for asymptomatic athletes who have tested positive for COVID-19 [Figure 1].

Athletes who experience mild symptoms but do not require hospitalisation are recommended two additional weeks of social distancing, followed by an evaluation of their situation based on blood tests ( $\mathrm{C}$-reactive protein, brain natriuretic peptide and troponin) and 12-lead electrocardiogram and echocardiography, if clinically indicated. A gradual return to exercise under the guidance of a medical team has also been recommended for this group [Figure 1], 5,8,12

A more complex investigation protocol is recommended for athletes with moderate to severe symptoms who needed hospitalisation. Those who were hospitalised with respiratory symptoms and myocardial injury are advised to undergo a comprehensive and multidisciplinary evaluation before they return to exercise [Figure 1]..$^{5,6,8,12}$

Ideally, this individual assessment should be done by a team comprising a sports physician, a sports cardiologist, a specialised pulmonologist and a professional athletic trainer. ${ }^{5,6}$ Barker-Davies et al. recommended an extensive exercise-abstinence period of three to six months in patients with confirmed myocarditis and that the adjustment of this period of rest should be based on the clinical severity and duration of the underlying disease. ${ }^{13}$ Verwoert et al. emphasised the need for a comprehensive rehabilitation plan including intensive monitoring for cardiac complications and a complete cardiovascular evaluation before return to exercise and sports. ${ }^{6}$ Overall, close monitoring of hospitalised athletes with COVID-19-related respiratory symptoms is warranted. It is also recommended that those with severe respiratory symptoms be monitored by a specialised pulmonologist even as they gradually return to exercise in keeping with how their recovery progresses. $^{8}$

\section{EXERCISE AND VIRAL INFECTIONS}

Almost all viral infections induce the recruitment and activation of inflammatory cells, such as macrophages and neutrophils, which release a wide range of molecules (cytokines, metalloproteinases and components of the oxygen burst) that have been associated with tissue damage or dysfunction. ${ }^{14}$ A good balance between protective immunity and inflammatory response facilitates both viral clearance and resolution of infection. Exercise acts as a modulator of the immune system, stimulating cellular immunity while reducing the risk of developing systemic inflammatory processes. ${ }^{15}$ Various studies have suggested that exercise is directly related to decreased mortality that may be caused by pneumonia and influenza, improvements in the cardiorespiratory function and metabolic profile, including those concerning triglycerides, cholesterol, fasting plasma glucose, blood pressure and waist circumference. .,9-11,16 $^{\text {, }}$ The additional potential benefits of exercise, especially exercise of low-to-moderate intensity, includes a reduction in respiratory infection rate and improved recovery rate following a viral infection. ${ }^{16}$

Apart from the positive effects of exercise on the overall cardiorespiratory and metabolic function, it has also been shown to alleviate various mental health issues as it reduces anxiety and depression while improving self-esteem and cognitive function. ${ }^{16}$ Recent studies have also shown that exercise relieves symptoms such as low self-esteem and mitigates negative effects of self-isolation during quarantine. ${ }^{15,16}$

Endogenous opioids such as endorphins play a significant role in the integration of hormonal and metabolic responses to exercise and are associated with euphoria, which has been shown to significantly increase after running or swimming. Improving COVID-19 infected patients' general health condition (physiological and psychological status) can facilitate their ability to carry out necessary activities of daily living and enable their return to work. ${ }^{16}$

As athletes of all levels return to exercise after a viral infection, special consideration should be given to the intensity and volume of exercise. For those who have been recovering from a COVID-19 infection, returning to exercise can be a challenge. In this context, return to exercise should be guided by clinical symptoms and symptomatic patients should be encouraged to follow a low-intensity exercise programme during the selfisolation after 72 hours of resolution of symptoms. This programme can be adjusted as per the available equipment such as a stationary bike and/or a treadmill or even include resistance exercises. ${ }^{8,17}$ High-intensity exercise should be avoided as it is associated with an increased risk of upper respiratory tract infection and 
significant complications and can even lead to sudden cardiac arrest. ${ }^{7,10,18}$ Previous studies show that low-tomoderate exercise improves immune function, while high-intensity exercise depresses the immune system and increases susceptibility to infection [Figure 2]. 7,10

It is important to note that the combination of highand low-intensity training is an important component for advanced athletes to elicit improvements in their aerobic capacity and achieve optimal performance when they exercise. ${ }^{19}$ A key concern for athletes is determining the optimal time before they can return to practicing and/or high-intensity exercise training following an infection. Returning to high-intensity exercise training while the body is still experiencing a systemic infection with persistent cough, fever and dyspnoea is strongly associated with a significant risk of major complications such as myocarditis. ${ }^{20}$

COVID-19-positive athletes should opt for a gradual return to exercise after the resolution of symptoms to achieve their pre-infection fitness level. ${ }^{7,8}$ While data related to the return to exercise after COVID-19 are limited, low-intensity exercise for one week is recommended before engaging in more strenuous and/or rigorous types of exercise. Due to the long period of physical inactivity (of at least two weeks of rest without exercising), those recovering from an infection may be at an increased risk of injury. ${ }^{8}$ To avoid such injuries, Barker-Davies et al. recommend three metabolic equivalents (METs) for a period of two to three weeks. ${ }^{13}$ Physical activity is classified as low-intensity (<3 METs), moderate-intensity (3-6 METs) and high-intensity (>6 METs). ${ }^{21,22}$ Exercising on a treadmill (walking velocity of $3.2 \mathrm{~km} / \mathrm{h}$ ), stationary bike (<50 Watts) and/or resistance exercise with light weights are equivalents of 3 METs and, hence, are possible options for starting an exercise programme. ${ }^{22}$ An exercise programme should be initiated at a low intensity which could be gradually made more rigorous [Figure 1]. ${ }^{21,22}$

An otherwise healthy athlete, infected with COVID-19, who underwent a self-limiting infection after being asymptomatic for seven days may return to exercise at $50 \%$ of normal intensity and volume. ${ }^{8}$ It should be noted that the current recommendations for physical activity in healthy individuals specify at least 150 minutes per week (30 minutes per day for five days) of moderate-intensity exercise or 75 minutes per week (25 minutes per day for three days) of vigorousintensity exercise or a combination of moderateand vigorous-intensity exercise. ${ }^{21}$ It is important to note that any applied exercise programme must be individualised according to the severity of the disease, and sometimes even a gradual return to exercise may take months.
Many athletes are reporting COVID-19-related symptoms after resolution of the infection, especially during high-intensity exercise. ${ }^{7,20}$ These may include the runner who experiences persistent fatigue and the swimmer who reports ongoing respiratory symptoms (cough and dyspnoea). ${ }^{8}$ It is important to note that exercise should be discontinued and a sports physician consulted in cases where symptoms such as fever, dyspnoea, cough, tachycardia and extreme fatigue are manifested as they may be associated with the recurrence or reactivation of the COVID-19 infection. $^{23}$ Additionally, it must be emphasised that initiating exercise at regular intensity and volume before complete recovery can increase the risk of serious injuries and/or illnesses. ${ }^{7}$ A potentially increased risk for illness in periods of high-intensity exercise is predominantly a concern for non-competitive, recreational athletes. ${ }^{19}$ It is expected that most will fully recover following a progressive individualised exercise programme. ${ }^{6,7}$

\section{Conclusion}

The COVID-19 infection is a global pandemic which affects all segments of the population to varying degrees, ranging from a few days of mild symptoms to myocardial injury, respiratory distress and death; athletes are not exempt from the threat of this infection. Healthcare professionals are faced with unprecedented challenges concerning the COVID-19 infection and the complications related to it. It is critical, therefore, to track and evaluate the rapidly evolving scientific landscape of returning safely to exercise after the COVID-19 infection. The development of elemental recommendations about the safe return of athletes to exercise is warranted.

\section{AUTHORS' CONTRIBUTION}

AVV and VB conceptualised and designed the data. Both authors were involved in drafting the manuscript. Both authors approve the final version of the manuscript.

\section{References}

1. Cucinotta D, Vanelli M. WHO declares COVID-19 a pandemic. Acta Biomed 2020; 91:157-60. https://doi.org/10.23750/abm. v91i1.9397.

2. Jacobs JJL. Persistent SARS-2 infections contribute to long COVID-19. Med Hypotheses 2021; 149:110538. https://doi. org/10.1016/j.mehy.2021.110538.

3. Araújo CG, Scharhag J. Athlete: A working definition for medical and health sciences research. Scand J Med Sci Sports 2016; 26:4-7. https://doi.org/10.1111/sms.12632. 
4. Yanguas X, Dominguez D, Ferrer E, Florit D, Mourtabib Y, Rodas G. Returning to sport during the Covid-19 pandemic: The sports physicians' role. Apunts Sports Med 2020; 55:49-51. https://doi.org/10.1016/j.apunsm.2020.06.001.

5. Löllgen H, Bachl N, Papadopouloou T, Shafik A, Holloway G, Vonbank K, et al. Infographic. Clinical recommendations for return to play during the COVID-19 pandemic. Br J Sports Med 2020; 55:344-5. https://doi.org/10.1136/bjsports-2020-102985.

6. Verwoert GC, de Vries ST, Bijsterveld N, Willems AR, vd Borgh R, Jongman JK, et al. Return to sports after COVID-19: A position paper from the Dutch Sports Cardiology Section of the Netherlands Society of Cardiology. Neth Heart J 2020; 28:391-5. https://doi.org/10.1007/s12471-020-01469-z.

7. Woods JA, Hutchinson NT, Powers SK, Roberts WO, GomezCabrera MC, Radak Z, et al. The COVID-19 pandemic and physical activity. Sports Med Health Sci 2020; 2:55-64. https:// doi.org/10.1016/j.smhs.2020.05.006

8. Metzl JD, McElheny K, Robinson JN, Scott DA, Sutton KM, Toresdahl BG. Considerations for return to exercise following mild-to-moderate COVID-19 in the recreational athlete. HSS J 2020; 16:1-6. https://doi.org/10.1007/s11420-020-09777-1.

9. Booth FW, Roberts CK, Laye MJ. Lack of exercise is a major cause of chronic diseases. Compr Physiol 2012; 2:1143-211. https://doi.org/10.1002/cphy.c110025.

10. Gentil P, de Lira CAB, Souza D, Jimenez A, Mayo X, de Fátima Pinho Lins Gryschek AL, et al. Resistance training safety during and after the SARS-Cov-2 outbreak: Practical recommendations. Biomed Res Int 2020; 2020:3292916. https:// doi.org/10.1155/2020/3292916.

11. Krogh-Madsen R, Thyfault JP, Broholm C, Hartvig Mortensen O, Olsen RH, Mounier R, et al. A 2-wk reduction of ambulatory activity attenuates peripheral insulin sensitivity. J Appl Physiol (1985) 2010; 108:1034-40. https://doi.org/10.1152/japplphysio 1.00977.2009.

12. Phelan D, Kim JH, Chung EH. A game plan for the resumption of sport and exercise after Coronavirus Disease 2019 (COVID-19) infection. JAMA Cardiol 2020; 5:1085-6. https:// doi.org/10.1001/jamacardio.2020.2136.

13. Barker-Davies RM, O'Sullivan O, Senaratne KPP, Baker P, Cranley M, Dharm-Datta S, et al. The Stanford Hall consensus statement for post-COVID-19 rehabilitation. Br J Sports Med 2020; 54:949-59. https://doi.org/10.1136/bjsports-2020-102596.
14. Rouse BT, Sehrawat S. Immunity and immunopathology to viruses: What decides the outcome? Nat Rev Immunol 2010; 10:514-26. https://doi.org/10.1038/nri2802.

15. Lega S, Naviglio S, Volpi S, Tommasini A. Recent insight into SARS-CoV2 immunopathology and rationale for potential treatment and preventive strategies in COVID-19. Vaccines (Basel) 2020; 8:224. https://doi.org/10.3390/vaccines8020224.

16. da Silveira MP, da Silva Fagundes KK, Bizuti MR, Starck É, Rossi RC, de Resende e Silva DT. Physical exercise as a tool to help the immune system against COVID-19: An integrative review of the current literature. Clin Exp Med 2021; 21:15-28. https://doi.org/10.1007/s10238-020-00650-3.

17. Toresdahl BG, Asif IM. Coronavirus Disease 2019 (COVID-19): Considerations for the competitive athlete. Sports Health 2020; 12:221-4. https://doi.org/10.1177/1941738120918876.

18. Baggish AL, Levine BD. Icarus and sports after COVID 19: Too close to the sun? Circulation 2020; 142:615-17. https://doi. org/10.1161/CIRCULATIONAHA.120.048335.

19. Hull JH, Loosemore M, Schwellnus M. Respiratory health in athletes: Facing the COVID-19 challenge. Lancet Respir Med 2020; 8:557-8. https://doi.org/10.1016/S2213-2600(20)30175-2.

20. Wilson MG, Hull JH, Rogers J, Pollock N, Dodd M, Haines J, et al. Cardiorespiratory considerations for return-to-play in elite athletes after COVID-19 infection: A practical guide for sport and exercise medicine physicians. Br J Sports Med 2020; 54:1157-61. https://doi.org/10.1136/bjsports-2020-102710.

21. González K, Fuentes J, Márquez JL. Physical inactivity, sedentary behavior and chronic diseases. Korean J Fam Med 2017; 38:111-15. https://doi.org/10.4082/kjfm.2017.38.3.111.

22. Pate RR, Pratt M, Blair SN, Haskell WL, Macera CA, Bouchard C, et al. Physical activity and public health. A recommendation from the Centers for Disease Control and Prevention and the American College of Sports Medicine. JAMA 1995; 273:402-7. https://doi.org/10.1001/jama.273.5.402.

23. Gousseff M, Penot P, Gallay L, Batisse D, Benech N, Bouiller K, et al. Clinical recurrences of COVID-19 symptoms after recovery: Viral relapse, reinfection or inflammatory rebound? J Infect 2020; 81:816-46. https://doi.org/10.1016/j.jinf.2020.06.073. 